\title{
Variabilité du panache turbide de la Gironde par télédétection. Effets des facteurs climatiques
}

\author{
Jean-Marie FROIDEFOND ${ }^{\text {a }}$, Anne-Marie JEGOU ${ }^{b}$, Julio HERMIDA a ${ }^{\text {, Pascal LAZURE }}{ }^{\text {, }}$, \\ Patrice CASTAING ${ }^{\text {a }}$ \\ ${ }^{a}$ Département de Géologie et Océanographie / UMR EPOC 5805-CNRS, Université Bordeaux-1, avenue des Facultés, \\ 33405 Talence cedex, France \\ ${ }^{\mathrm{b}}$ IFREMER, (DEL/LHS), BP 70, 29280 Plouzané, France
}

(Revised 08/01/98, accepted 09/01/98)

\begin{abstract}
Variability of the Gironde turbid plume by remote sensing. Effects of climatic factors. The Gironde estuary (France) discharges to the ocean an important amount of suspended particulate matter in the form of turbid plumes. The surface plume is more particularly studied from coastal oceanographic surveys and NOAA/AVHRR satellite data collected during a French programme of coastal oceanography (PNOC-Atlantic). The AVHRR reflectances are atmospherically corrected according to an algorithm based on the clear water concept. The comparison with suspended sediment concentrations are realised by direct and indirect calibrations. The correlations obtained show that at a same concentration the reflectance varies, probably because of varying particle (floc) size and composition. The shape and the surface measured from low resolution $(4 \mathrm{~km} \times 4 \mathrm{~km})$ and high resolution $(1 \mathrm{~km} \times 1 \mathrm{~km})$ AVHRR data are then compared to the forcings introduced by the fluvial output, the tide, neap and spring tide and the wind variations. The latter have an important effect on the orientation of the distal part of the turbid plume. (C) Elsevier, Paris
\end{abstract}

\section{turbid plume / remote sensing / hydrology / climatology / Gironde}

Résumé - L'estuaire de la Gironde (France) expulse vers l'océan une quantité importante de particules sédimentaires en suspension sous forme de panaches turbides. Le panache de surface est plus particulièrement étudié à partir de missions océanographiques côtières et de données satellitales NOAA-AVHRR acquises dans le cadre du Programme National d'Océanographie Côtière PNOC-Atlantique. Celles-ci sont corrigées des effets atmosphériques suivant un algorithme basé sur le concept des eaux claires. Puis la comparaison des réflectances avec les données in situ est réalisée par étalonnage direct et indirect. Les corrélations obtenues montrent que pour une même concentration, la réflectance varie vraisemblablement suivant la taille et la composition des particules. Ensuite, la forme et l'extension du panache mesurées à partir de données AVHRR à basse résolution $(4 \mathrm{~km} \times 4 \mathrm{~km})$ et à haute résolution $(1 \mathrm{~km} \times 1 \mathrm{~km})$ sont comparées aux forçages introduits par le débit fluvial, la marée, la variation du coefficient de marée (vives-eaux et mortes-eaux) et le vent. Celuici a un effet très important sur l'orientation de la partie distale de la plume. (C) Elsevier, Paris

\section{panache turbide / télédétection / hydrologie / climatologie / Gironde}

\section{INTRODUCTION}

Les sédiments en suspension apportés par les fleuves (particules terrigènes) ont un effet important sur les eaux côtières. Ils modifient la pénétration de la lumière et par conséquent la productivité primaire. Ils servent de support à certains nutriments et à de nombreuses bactéries. Ils peuvent transporter des métaux lourds, tel que le cadmium, un polluant important de l'estuaire de la Gironde. Aussi est-il nécessaire de quantifier la variabilité des

\footnotetext{
* Correspondence and reprints
} 
panaches terrigènes expulsés par les fleuves pour surveiller l'écosystème côtier soumis à l'accroissement des activités humaines. La télédétection spatiale est un moyen bien adapté pour atteindre cet objectif.

Plusieurs articles ont été publiés au cours de ces dernières années pour établir des relations entre les concentrations en particules terrigènes et les données satellitales des capteurs LANDSAT $[4,17,19]$ et SPOT $[10,18]$. Mais ces satellites ont un délai trop important entre deux prises de vue pour évaluer la variabilité d'un panache turbide. Le capteur AVHRR sur les satellites NOAA était le mieux adapté puisqu'il donne plusieurs images par jour, exploitables si le ciel est sans nuage. Ainsi, Stumpf et Pennock [20] ont montré qu'il permettait de connaître la répartition des concentrations en Matière En Suspension (MES). En se basant sur les données AVHRR et sur les données hydrologiques, il est possible de comparer la variabilité du panache turbide de surface de la Gironde avec les effets des facteurs climatiques (débits fluviaux, marée, vent).

\section{DESCRIPTION DE LA ZONE D'ÉTUDE}

La zone d'étude comprend le proche plateau situé à l'ouest de l'estuaire de la Gironde dans le golfe de Gascogne (figure 1). La Gironde qui réunit les fleuves Garonne et Dordogne est I'un des plus grands estuaires des côtes européennes. Les deux fleuves drainent un bassin de $71000 \mathrm{~km}^{2}$. Le débit moyen annuel d'eau douce est de $900 \mathrm{~m}^{3} \cdot \mathrm{s}^{-1}$. Le débit mensuel moyen varie très fortement de l'hiver $\left(1450 \mathrm{~m}^{3} \cdot \mathrm{s}^{-1}\right.$ en janvier $)$ à l'été $\left(235 \mathrm{~m}^{3} \cdot \mathrm{s}^{-1}\right.$ en août) avec des crues exceptionnelles atteignant $7500 \mathrm{~m}^{3} \cdot \mathrm{s}^{-1}$. Les effets des débits fluviaux sont modulés par la marée de type macro-tidal. A l'embouchure de l'estuaire, l'amplitude de la marée dépasse 5,5 m durant les marées de vives-eaux et les courants de marée peuvent dépasser $3 \mathrm{~m} \cdot \mathrm{s}^{-1}$ en surface [6].

L'estuaire a une longueur de $100 \mathrm{~km}$ environ. Il transporte une quantité importante MES évaluée à 2,2 millions de tonnes par an dont 1,5 million de tonnes en suspension et 0,7 millions de tonnes par charriage [2] et des substan-

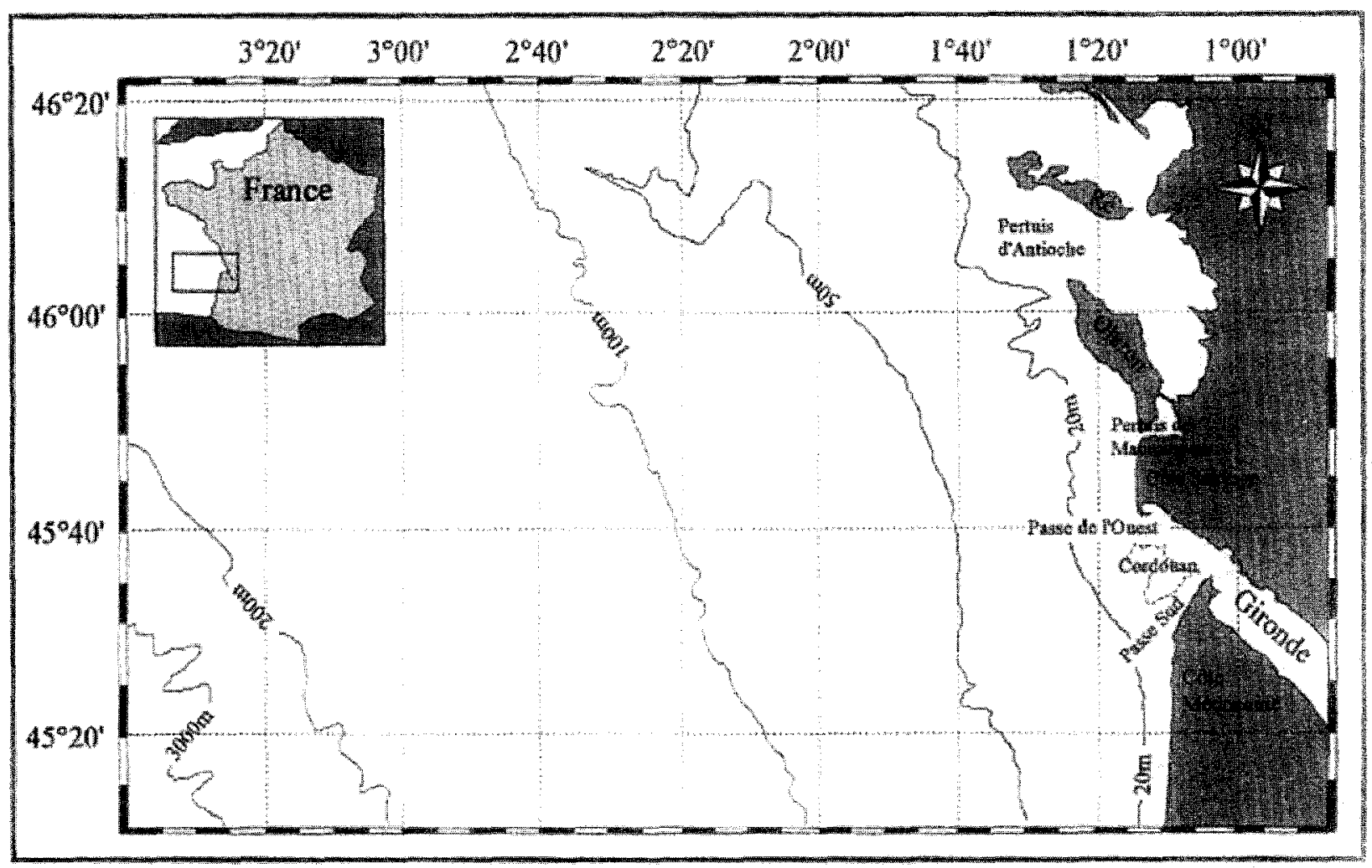

Figure 1. La zone d'étude avec la Gironde et le plateau continental Aquitain.

Figure 1. The study area with the Gironde estuary and the Aquitaine continental shelf. 
ces dissoutes. En particulier, il transporte un polluant, le cadmium, qui est un métal lourd provenant d'anciennes mines de zinc. Ce cadmium est apporté à l'océan sous forme dissoute $(2 / 3)$ et sous forme particulaire (1/3).

Sur le proche plateau continental, la distribution de taille des particules sédimentaires est bimodale, avec un premier mode à 7,2 micromètres (partie minérale essentiellement) et un second à 15 micromètres (partie biogénique en majorité) d'après des mesures sur les particules désagrégées [23]. Sans désagrégation, le diamètre moyen des agrégals (flucons) est situé entre 100 el 500 micromètres [8]. Ces particules en suspension sont composées de quartz, de micas, de minéraux argileux et de composés organiques. Dans l'embouchure de la Gironde, la concentration en carbone organique dissous varie entre 1 et 2 mg. $l^{-1}[16]$. Les concentrations en phytoplancton dépassent rarement $10 \mathrm{mg} \cdot \mathrm{m}^{-3}$. Elles sont le plus souvent comprises entre 1 et $3 \mathrm{mg} \cdot \mathrm{m}^{-3}$.

La morphologie de l'embouchure de la Gironde est caractérisée par un plateau rocheux, le plateau de Cordouan, qui sépare deux passes : la passe Sud, de 10 à $20 \mathrm{~m}$ de profondeur, et la passe de l'Ouest (chenal de navigation), de 15 à $30 \mathrm{~m}$ de profondeur, avec des ridins sur le fond. Le plateau interne présente une pente faible de $1,5 \mathrm{~m}$ par kilomètre. Il est couvert de sables fins et grossiers excepté entre $-30 \mathrm{~m}$ et $-60 \mathrm{~m}$ où apparaissent deux zones de dépôts de vases toujours actives : les vasières ouest et sud Gironde. Ce proche plateau reçoit aussi une partie des débits fluviaux de la Charente et de la Seudre qui sont expulsés par le Pertuis de Maumusson entre l'Ile d'Oléron et la côte, à $15 \mathrm{~km}$ au nord de l'embouchure de la Gironde.

Lcs courants de marce sont des facteurs hydrodynamiques importants dans les embouchures de la Gironde et du Pertuis de Maumusson. Le long de la côte aquitaine, les vents dominants sont de secteur ouest. Ils dépassent une ou plusieurs fois par an $100 \mathrm{~km} \cdot \mathrm{h}^{-1}$. Les houles provenant du secteur ouest sont parfois importantes et peuvent dépasser $10 \mathrm{~m}$ d'amplitude.

\section{DONNÉES}

Trois types de données sont utilisés pour connaître le panache turbide de la Gironde et sa variabilité spatio-temporelle: 1) des données hydrologiques, 2) des données climatologiques, 3) des données satellitales AVHRR/ NOAA, pour la plupart acquises dans le cadre du Programme National d'Océanographie Côtière PNOC-Atlantique [15].

\subsection{Données hydrologiques et optiques}

Avec lc démarrage du programme PNOC-Atlantique des missions océanographiques furent réalisées sur le plateau ouest-Gironde à différentes saisons et au cours de situations hydrologiques contrastées comme le montre le tableau $I$.

Les données hydrologiques de température, conductivité (salinité), oxygène dissous, turbidité (néphélomètre OBS-3), fluorescence (fluorimètre Aquatracka), pénétra-

Tableau I. Missions océanographiques dont les données sont utilisées dans cette étude. Les températures et les salinités de surface sont inscrites en se déplaçant de l'embouchure vers le plateau externe.

Table I. Oceanographic surveys whose data are used in this study. Surface temperatures and salinities are inscribed from the mouth to the outer shelf.

\begin{tabular}{|c|c|c|c|c|}
\hline Missions & Dates & $\begin{array}{l}\text { Températ. } \\
\text { de surface }\end{array}$ & $\begin{array}{l}\text { Salinités* } \\
\text { de surface }\end{array}$ & $\begin{array}{l}\text { MES de surface } \\
(\mathrm{mg} / \mathrm{L})\end{array}$ \\
\hline Ecomarge 92 & Mai 92 & $13,7-12,8-13,1-\cdots$ & 22,2-33,6-34,7---- & $87-33-1,5-\cdots$ \\
\hline PNOCat-2 & Mars 93 & $10,8-10,8-10,6$ & $25,7-30,9-35,2$ & $33-17-3,8--$ \\
\hline PNOCat-3 & Avril 93 & $--11,8-12,3-\cdots$ & $---33,5-34,7-\cdots$ & - \\
\hline Calibsat-1 & Mai 93 & -14,9-17,1-. & $--31,0-35,3$ & $\cdots 45-4,1-2,7-\cdots$ \\
\hline PNOCat-4 & juillet 93 & 20,4-19,8-19,5- & $26,0-31,0-32,4-35,5--$ & --o-n--- \\
\hline Calihsat -3 & Mars 94 & $9,5-9,9-10,0-12,4$ & $19,2-22-31-32,8-35,5$ & $57-65-8-3,8-2,4$ \\
\hline PNOCat-5 & Mai 94 & $14,4-14,5-13,4-13,9--$ & 22,2-30,0-34,3- & $14,5-17,0-4,1-3,2$ \\
\hline Calibsat-4 & Juillet 94 & $19,4-18,0-18,5-20,7$ & $21,8-31-33,5-35,0--$ & $20-7,5-2-1,3$ \\
\hline Biomet & Mai 95 & $--16,9-15,8-15,5$ & $---31,1-33,2-34,1---$ & - n-.-- \\
\hline
\end{tabular}


tion de la lumière (capteur PAR) furent obtenues avec une sonde multiparamètre Sea Bird (SBE-25) sur des profils verticaux et une sonde $\mathrm{ME}$ qui a été utilisée pendant les missions Ecomarge 92 et Biomet-1. Les couleurs de l'eau pour l'interprétation des images satellitales ont été mesurées par ciel clair avec un radiomètre CE-310 (Cimel) à trois canaux $(500-600 \mathrm{~nm}, 600-700 \mathrm{~nm}$ et $800-900 \mathrm{~nm})$. Les matières particulaires en suspension furent échantillonnées avec des bouteilles hydrologiques à différentes profondeurs. Les concentrations furent mesurées par filtration et pesée [13].

\subsection{Données climatologiques et débits fluviaux}

Les débits fluviaux sont mesurés par le Port Autonome de Bordeaux (PAB) sur les deux principaux fleuves, la Garonne et la Dordogne. Les hauteurs de la marée ont été relevées sur les annuaires du PAB. Les vitesses et les directions de vents ont été fournies par Meteo France (base de données climatologiques constituée par IFREMER pour le PNOC). Ces données proviennent du sémaphore de Chassiron situé sur la pointe Nord de l'Tle d'Oléron.

\subsection{Données satellitales AVHRR/NOAA-11}

Le capteur AVHRR (Advanced Very High Resolution Radioneter) sur le satellite NOAA-11 a été choisi pour les raisons suivantes : il passait chaque jour entre $13 \mathrm{~h}$ et $16 \mathrm{~h}$ au-dessus du site d'étude ; il enregistrait simultanément des images dans le visible et l'infrarouge thermique.

Les bandes spectrales du capteur AVHRR utilisables pour mesurer les turbidités de surface sont les suivantes :

Image du canal 1 (C1) bande spectrale : $580 \mathrm{~nm}-680 \mathrm{~nm}$ Image du canal $2(\mathrm{C} 2)$ bande spectrale : $730 \mathrm{~nm}-1100 \mathrm{~nm}$

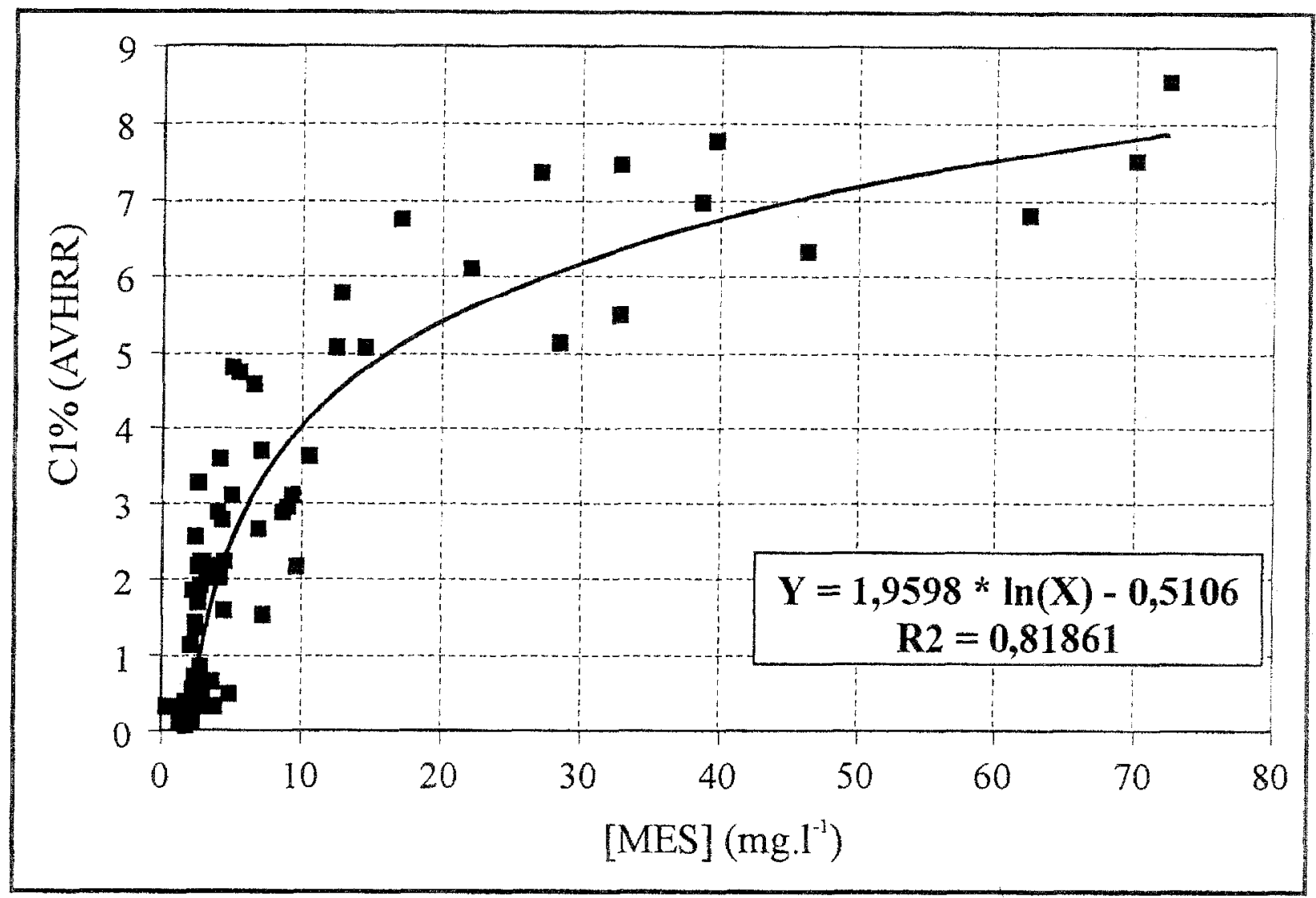

Figure 2. Etalonnage direct. Relation entre les concentrations en sestons et les réflectances mesurées par le capteur AVHRR (correction C1 « eaux claires $»$ ).

Figure 2. Direct calibration. Relationship between the seston concentrations and the reflectances measured with the AVHRR/NOAA sersor (corrected C1 - 'C1 clear water'). 
Deux types de données numériques fournis par le Centre de Météorologie Spatiale de Lannion (CMS, MétéoFrance) ont été acquises et traitées : 1) une trentaine de scènes à résolution maximale $(1 \mathrm{~km} \times 1 \mathrm{~km}$ à la verticale du satellite) sous forme d'images $\mathrm{C} 1$ et $\mathrm{C} 2$ représentant une surface de $1500 \mathrm{~km}$ par $2500 \mathrm{~km}$ prises au cours des missions océanographiques. 2) des données journalières à basse résolution $(4 \mathrm{~km} \times 4 \mathrm{~km}$ ), sous forme d'imagettes C1 et C2 pour les années 1992 et 1993. Sur la totalité des 730 scènes, le couvert nuageux n'a permis d'en exploiter que 110.

\section{MÉTHODE D'TNTERPRÉTATION DES DONNÉES SATELLITALES}

La méthode d'interprétation des données satellitales en vue de la quantification des MES comprend les étapes de correction atmosphérique et d'étalonnage avec les mesures in situ.

\subsection{Correction atmosphérique des images satellitales}

Les radiances enregistrées dans chaque bande spectrale (image numérique) sont captées sous forme de valeurs entières sur 10 bits par le centre d'acquisition du CMS, puis converties en réflectances extra-atmosphériques (albédos) et codées sur 16 bits. Ces valeurs furent ensuite corrigées par le CMS des effets atmosphériques dus à l'absorption et à la diffusion moléculaire. Nous les avons reçues sous forme de scènes numériques à pleine résolution et à basse résolution. Mais des corrections atmosphériques complémentaires étaient indispensables pour comparer ces données.

En effet l'atmosphère modifie les réflectances (lumière réfléchie et rétrodiffusée) de la surface de l'eau en raison des molécules gazeuses (azote, oxygène, gaz carbonique, ozone) et des aérosols secs et humides qu'elle contient. En schématisant, la réflectance au sommet de l'atmos-

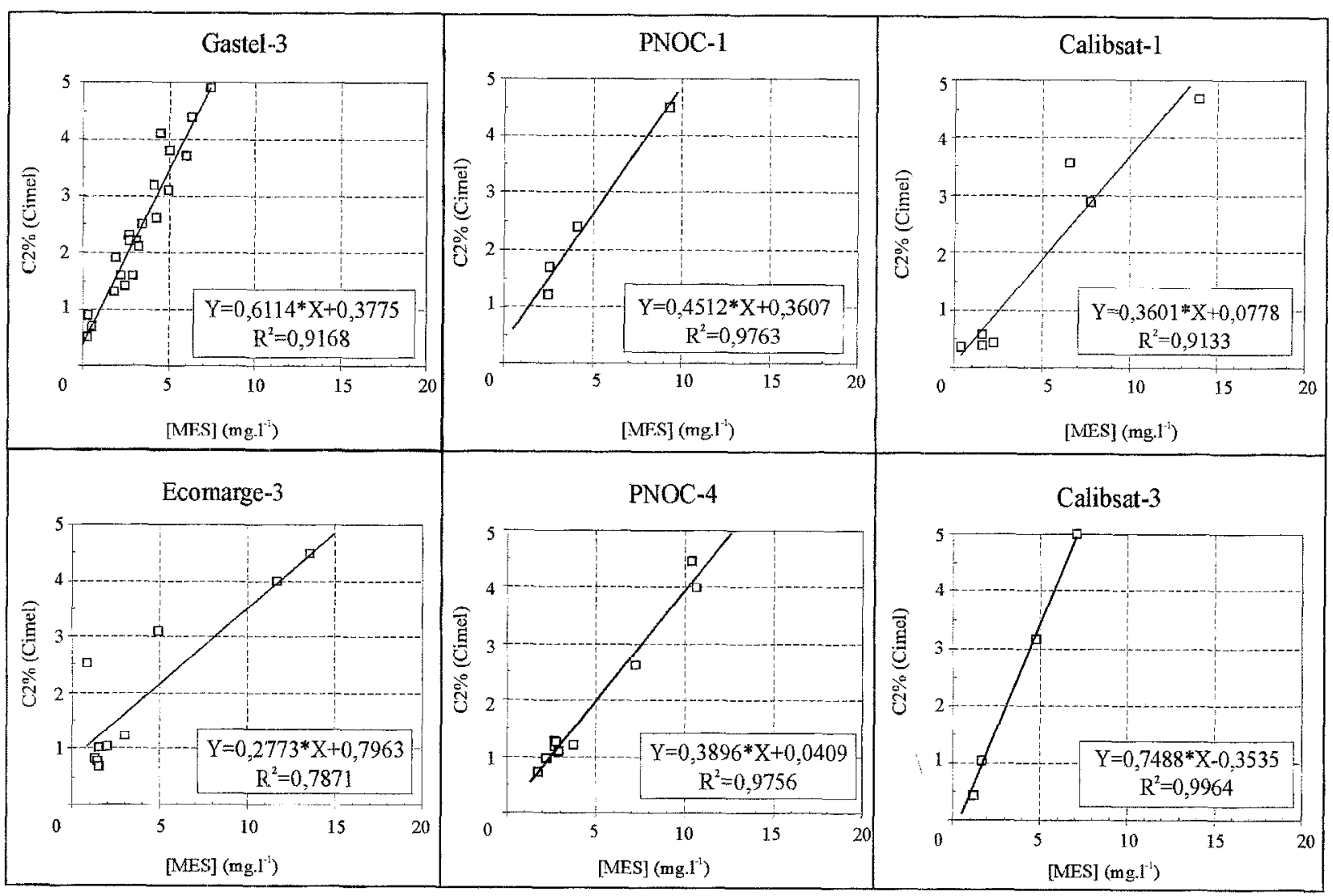

Figure 3. Étalonnage indirect avec un radiomètre portable. Relations entre les réflectances mesurées juste au-dessus de l'océan et les concentrations en sestons pour plusieurs missions océanographiques.

Figure 3. Indirect calibrations with a portable radiometer. Relationships between reflectances measured just above sea water and seston concentrations during oceanographic surveys. 
phère $\left(\rho^{*}\right)$ est égale à la somme de la réflectance des aérosols, ( $\rho$ aérosols) des molécules gazeuses ( $\mathrm{r}$ mol) et de l'eau ( $\rho w)$ multipliée par la transmission diffuse (Td),

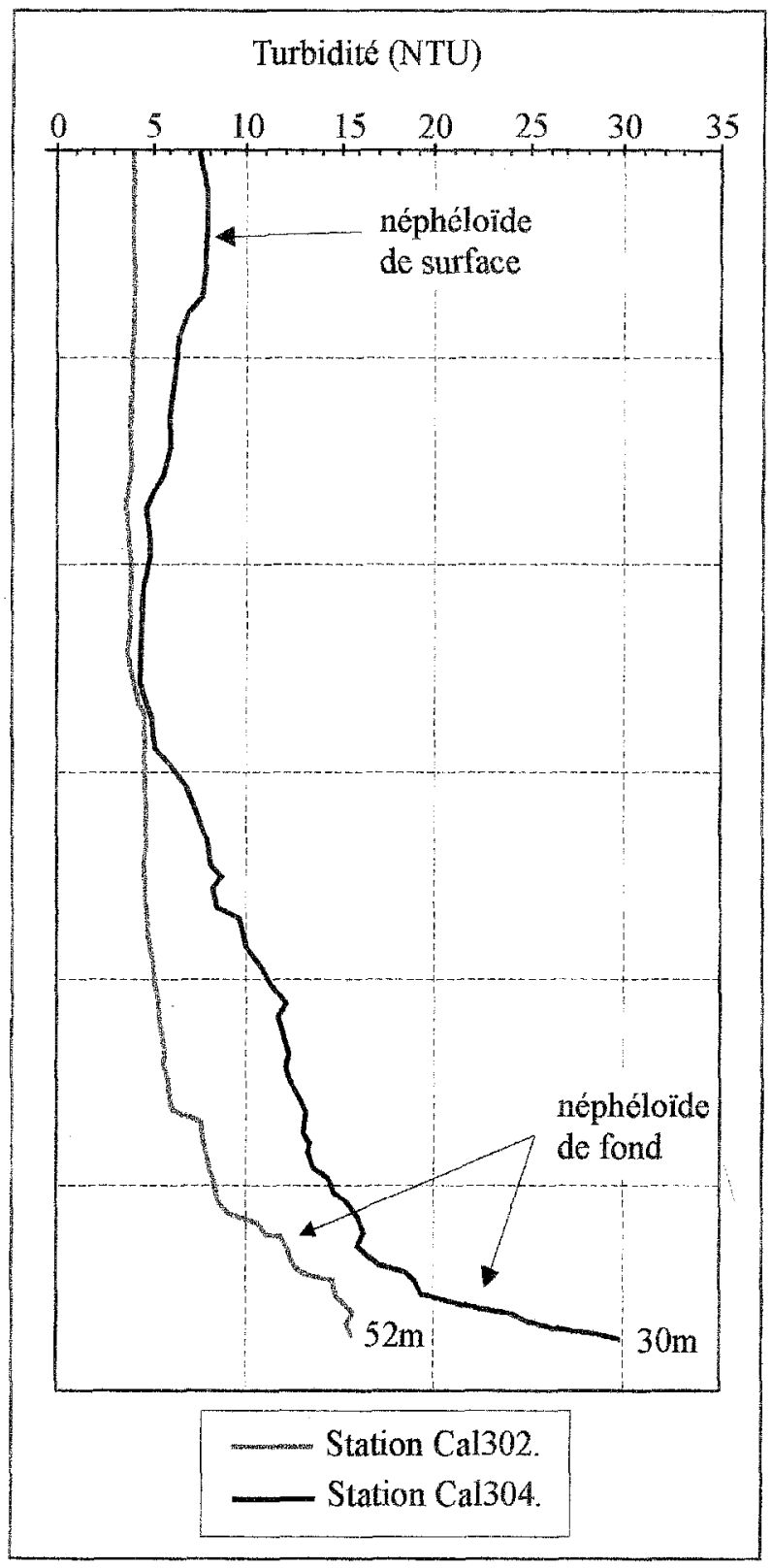

Figure 4. Profils verticaux représentant les variations de turbidité à l'extérieur de l'embouchure (fonds de $30 \mathrm{~m}$ ) et plus au large (fonds de $50 \mathrm{~m}$ ).

Figure 4. Vertical profiles showing turbidity variations on the outer part of the Gironde mouth $(30 \mathrm{~m}$ depth) and on the shelf (50 $\mathrm{m}$ depth). l'ensemble multiplié par la transmission gazeuse (Tg) sul. vant la relation simplifiée:

$r^{*}=(\rho \cdot a e r o+p \cdot m o l+\rho w \cdot T d) \cdot T g$

Les réflectances enregistrées par le capteur AVHRR dans les canaux $1(\mathrm{C} 1)$ et $2(\mathrm{C} 2)$ proviennent pour $10 \%$ à $20 \%$ de l'eau et pour $80 \%$ à $90 \%$ de l'atmosphère. La correction atmosphérique a pour but d'éliminer cette contribution atmosphérique afin de ne conserver que la réflectance de la surface de l'eau.

Les données fournies par le CMS sont seulement corrigées des effets dus aux molécules (réflectance de Rayleigh). Pour les corriger des teneurs en aérosols, nous avons procédé de la façon suivante. La réflectance « aérosol » est mesurée sur limage satellitale du canal 1 en extrayant la réflectance au dessus d'une zone située au large de la Gironde entre $80 \mathrm{~km}$ et $100 \mathrm{~km}$ des côtes où la concentration en MES est généralement inférieure à $1 \mathrm{mg} .1^{-1}$ (La réflectance de l'eau claire est considérée comme nulle dans le canal 1). Cette valeur est appelée « réflectance sur eau claire » $(\mathrm{R} \% \mathrm{ec})$ et correspond à la réflectance des aérosols si elle n'est pas nulle. Ensuite cette valeur est soustraite à tous les pixels de l'image. Les réflectanccs obtcnucs sont appclćes rćflectances normalisées :

$\mathrm{R} \%(\mathrm{C} 1)$ normalisées $=\mathrm{R} \%(\mathrm{C} 1)-\mathrm{R} \% \mathrm{ec}$

Cette réflectance $(\mathrm{R} \% \mathrm{ec})$, mesurée directement à partir des valeurs des pixels sur une zone d'eau claire adjacente à la zone d'étude, est différente pour chaque scène AVHRR. Par exemple, elle était de 3,16\% le 4 juillet 1989 , de $1,8 \%$ le 4 mars 1990 , de $2,7 \%$ le 5 mai 1990 . etc. De cette façon, toutes les images du canal 1 sont recalées par rapport à une cible de référence, « l'eau claire », et peuvent ainsi être comparées les unes aux autres [12]. Ponctuellement un traitement plus précis peut être appliqué [7].

En cas de scintillement solaire de la surface de l'eal ("sun-glitter"), la soustraction «image $\mathrm{Cl}$ - image C2 » est utilisée. En effet le scintillement affecte de façon presque identique les deux images. Par conséquent une soustraction des images $\mathrm{C} 1-\mathrm{C} 2$ efface en grande partie cet effet. Mais dans les eaux côtières où la turbidité dépasse fréquemment $15 \mathrm{mg} . \mathrm{l}^{-1}$, l'image $\mathrm{C} 2$ « enregistre » aussi cette turbidité ce qui entraîne une réduction artificielle des concentrations en MES que l'on cherche justement à mesurer. Par conséquent, cette dernière correction est seulement utilisée pour cartographier la structure du panache turbide (analyse qualitative). 


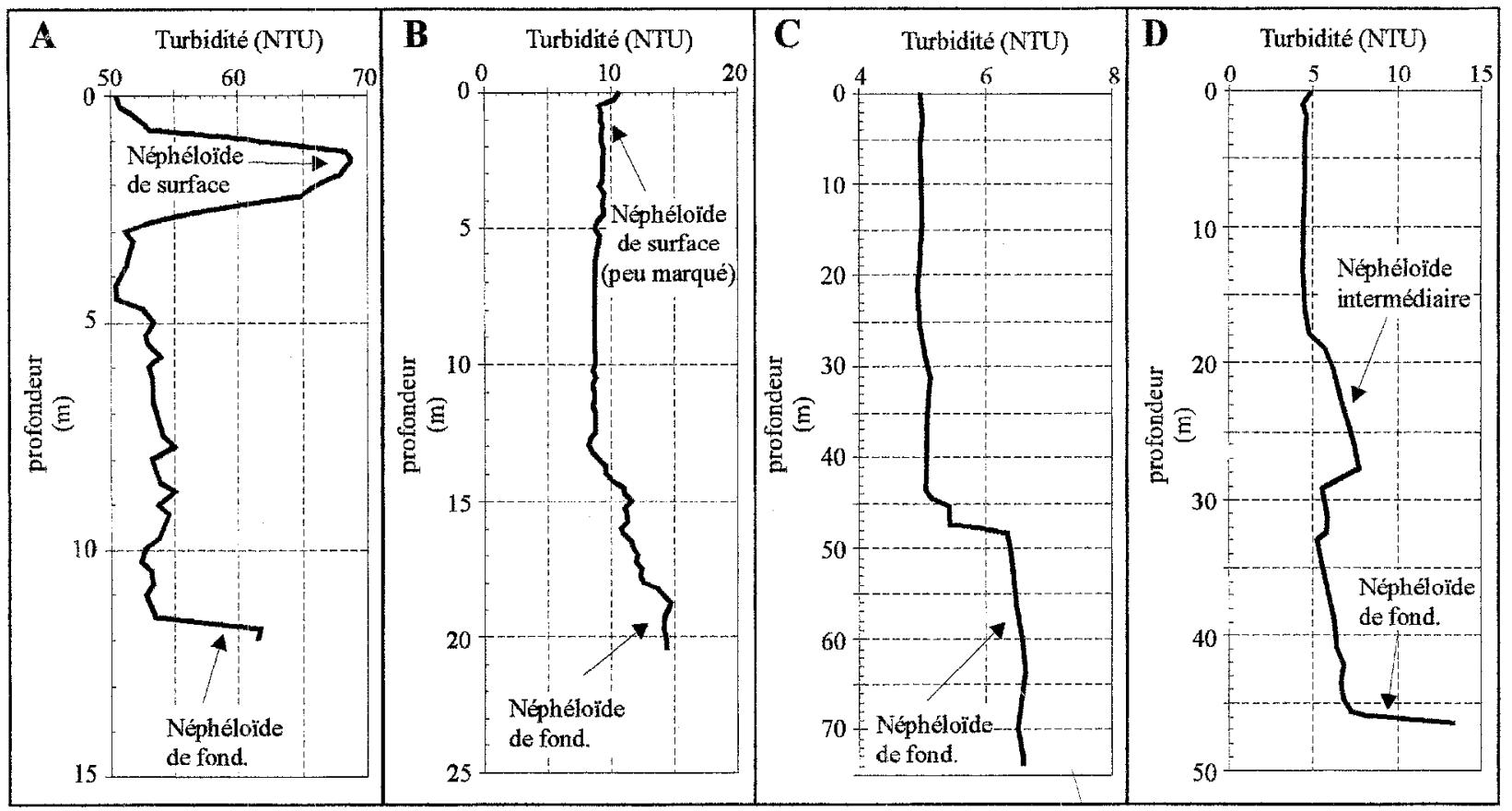

Figure 5. Profils verticaux enregistrés pendant plusieurs missions océanographiques représentant des néphéloïdes situés à différentes profondeurs.

Figure 5. Vertical profiles recorded during oceanographic surveys showing nepheloïd layers at different depths.

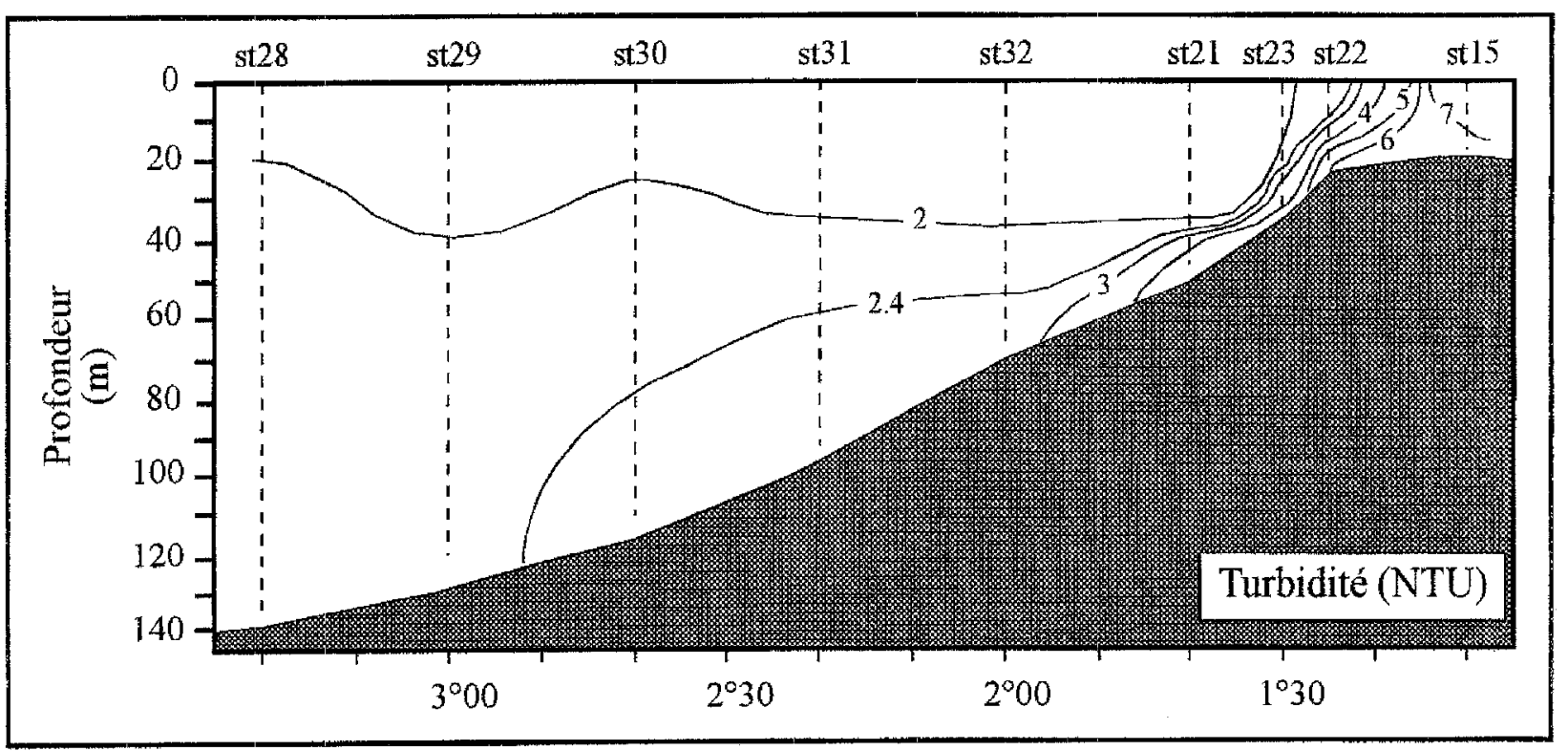

Figure 6. Les panaches turbides de sırface et de fond en période d'étiage. Coupe verticale orientée EW à la latitude $45^{\circ} 40^{\prime} \mathrm{N}$ durant la mission Calibsat-4 (juillet 1994).

Figure 6. Surface and bottom turbid plumes in low waler period. EW cross section at $45^{\circ} 40^{\prime} \mathrm{N}$ during the Calibsat-4 cruise (July 1994 ). 


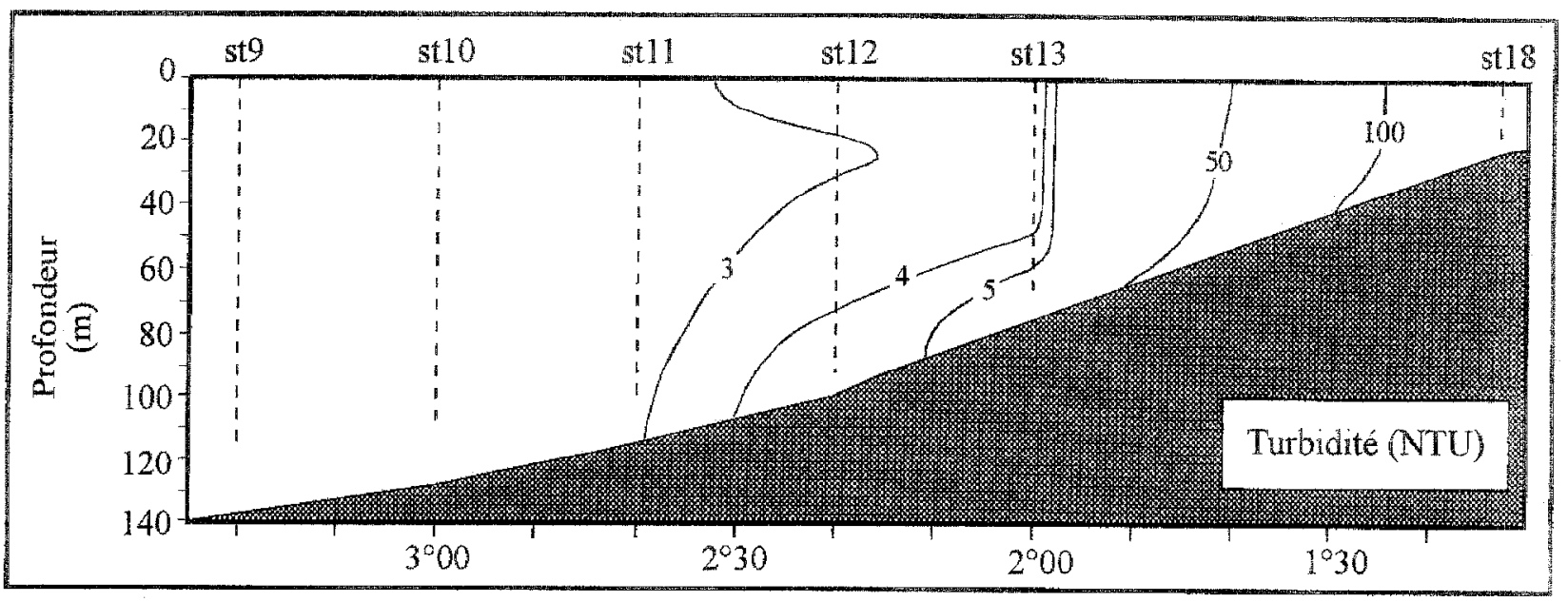

Figure 7. Les panaches turbides de surface et de fond en période de crue. Coupe verticale orientée EW à la latitude $45^{\circ} 40^{\prime} \mathrm{N}$ duran la mission Calibsat-3 (janvier 1994)

Figure 7. Surface and bottom plumes in flood period. EW cross section at $45^{\circ} 40^{\prime} \mathrm{N}$ during the Calibsat-3 cruise (January 1994$)$.

\section{2. Étalonnage : comparaison des concentrations en MES et des réflectances}

Pour établir une relation entre les conecntrations en MES et les données AVHRR deux procédures ont été mises au point et appliquées au cours de ces dernières années. Une calibration directe obtenue en comparant directement les données AVHRR avec les MES mesurées au cours des missions océanographiques et une calibration indirecte en comparant les MES avec les réflectances mesurées simultanément avec un radiomètre de terrain.

\subsection{1. Étalonnage direct : comparaison entre les concen- trations en MES el les données AVHRR}

Cet étalonnage est relativement difficile à obtenir puisqu'il faut impérativement se trouver en mer pendant l'acquisition satellitale et par beau temps. Lorsque ces conditions sont satisfaites, les points de localisation des mesures hydrologiques (MES) sont superposés sur l'image $\mathrm{Cl}$ de l'AVHRR. Ensuite les valeurs correspondantes sont reportées sur un diagramme. Seul les points situés à l'extérieur de l'embouchure sont pris en compte. En effet pour que l'étalonnage ait un sens, il faut supposer la stabilité des masses d'eau, ce qui est incompatible avec les courants de marée dans l'embouchure. Le diagramme obtenu (figure 2) montre une relation de type logarithmique lorsque les concentrations en MES varient de $0,5 \mathrm{mg} . \mathrm{l}^{-1}$ à $70 \mathrm{mg} \cdot \mathrm{l}^{-1}$. Si l'on ne considère que les faibles concentrations, cette relation apparaît linéaire [10, [1, 12].

\subsubsection{Etalonnage indirect : comparaison entre les concentrations en MES et les données radiométriques de. terrain}

Pour six missions océanographiques effectuées par mer calme et ciel clair, en mars 90 (Gastel 3), février 93 (Pnocat-1), avril 93 (Calibsat-1), juin 93 (Ecomarge 3), juillet 93 (Pnocat-4) et janvier 94 (Calibsat-3), la réflectance de la surface de l'eau a été mesurée avec un radiomètre CE-310, notamment dans la bande spectrale $600 \mathrm{~nm}$ $700 \mathrm{~nm}$, proche de celle du canal 1 de l'AVHRR (580 nm$680 \mathrm{~nm}$ ). Ces réflectances sont reportées en fonction des concentrations en MES correspondantes sur les diagrammes de la figure 3. Les droites d'ajustement ont des pentes très variables avec des valeurs comprises entre 0,28 et 0,75 qui peuvent s'expliquer par des facteurs tels que la variation de densité des particules (organiques ou minérales), la taille des agrégats et l'effet de réflectivité du ciel sur l'eau [13].

La comparaison des étalonnages directs et indirects (fig $\ell$ res 2,3) fait apparaitre une concordance relative pour les concentrations en MES inférieures à $10 \mathrm{mg} \cdot \mathrm{l}^{-1}$. Dans cette gamme, la relation est linéaire, en première approximation, avec une réflectance variant le plus souvent entre $0,5 \%$ et $5 \%$. La calibration indirecte est plus satisfaisante car les données sont acquises simultanément. Mais 


\section{Debit en $\mathrm{m} 3 / \mathrm{s}$}

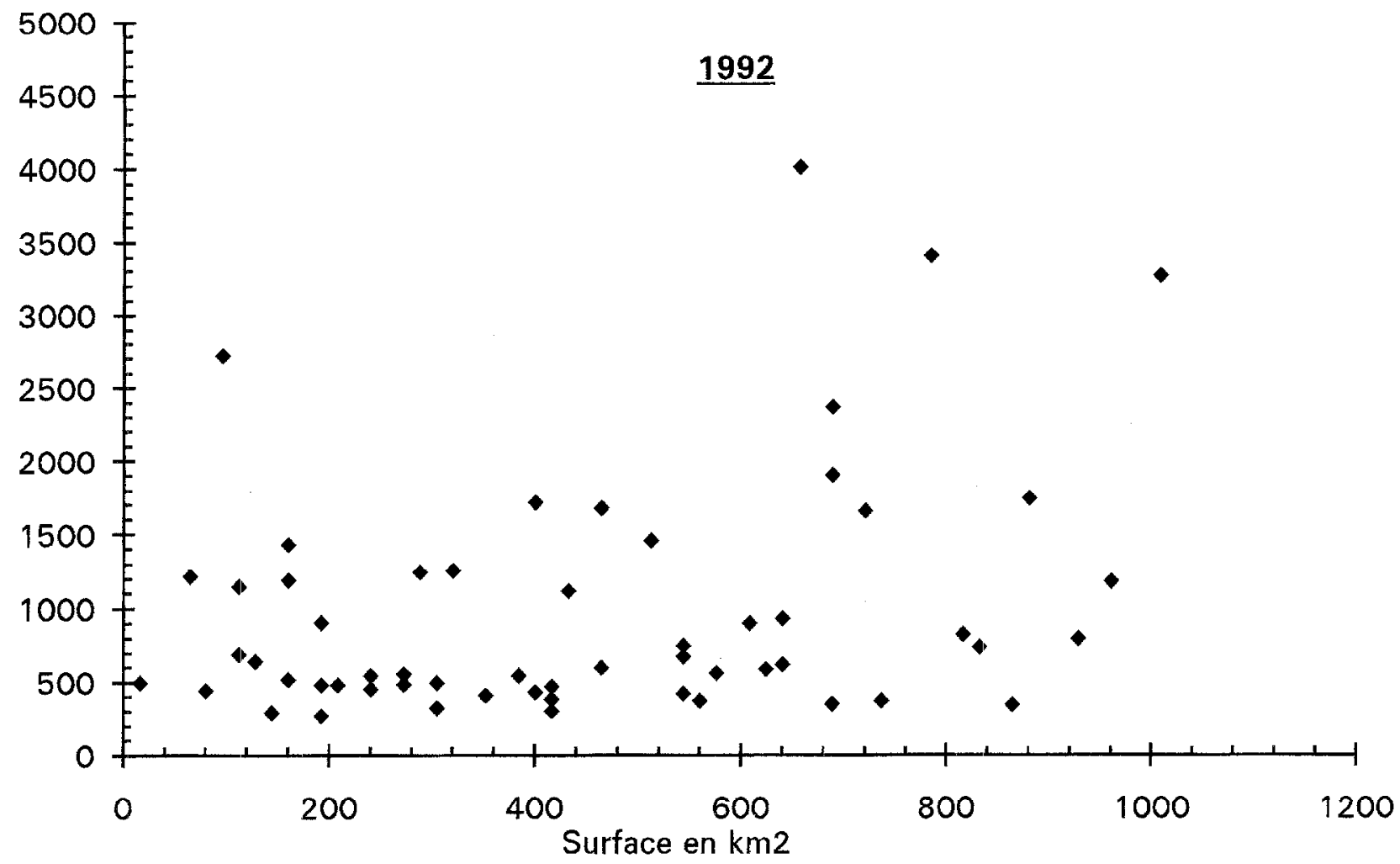

Figure 8. Variations de la surface du panache par rapport au débit fluvial en 1992.

Figure 8. Variations of the turbid plume areas in terms of fluvial outputs in 1992.

les caractéristiques des particules variant rapidement, ces mesures doivent être réalisées au moment de l'acquisition satellitale.

\section{RÉSULTATS}

\subsection{Position verticale des néphéloïdes dans l'embouchure et au large de la Gironde}

Différentes couches turbides (néphéloïdes) ont été repérées à partir des enregistrements verticaux du néphélomètre OBS-3. La disposition la plus fréquente est la suivante: à quelques kilomètres au large de l'embouchure, les eaux côtières présentent un néphéloïde de surface peu marqué et un néphéloïde de fond mieux individualisé (exemple du profil 1 enregistré par des fonds de $30 \mathrm{~m}$, figure 4). Sur le second profil (figure 4, profil 2 enregistré le même jour que le précédent) corres- pondant à une station plus au large (52 m) le néphéloïde de fond est encore visible, alors que celui de surface n'est plus perceptible [13].

Parfois, un néphéloïde de surface peut être très marqué dans l'embouchure (figure $5 a$ ). D'autres fois, la turbidité est homogène jusqu'à 3 ou $4 \mathrm{~m}$ du fond, puis elle augmente rapidement en individualisant un néphéloïde de fond (figure 5b). Dans un seul cas (mission PNOCat-3), un néphéloïde « intermédiaire » a été observé à mi-profondeur (figure 5d). Pendant les missions PNOCat-3 et PNOCat-5, la structure observée comportait une couche de surface homogène peu turbide et une couche inférieure homogène plus lurbide comme le montre la figure $5 c$.

\subsection{Panaches turbides de surface et de fond}

Les valeurs du néphélomètre (en unité instrumentale NTU) à $1 \mathrm{~m}$ sous la surface et à $2 \mathrm{~m}$ du fond ont été reportées sur plans cartographiques après chaque mis- 


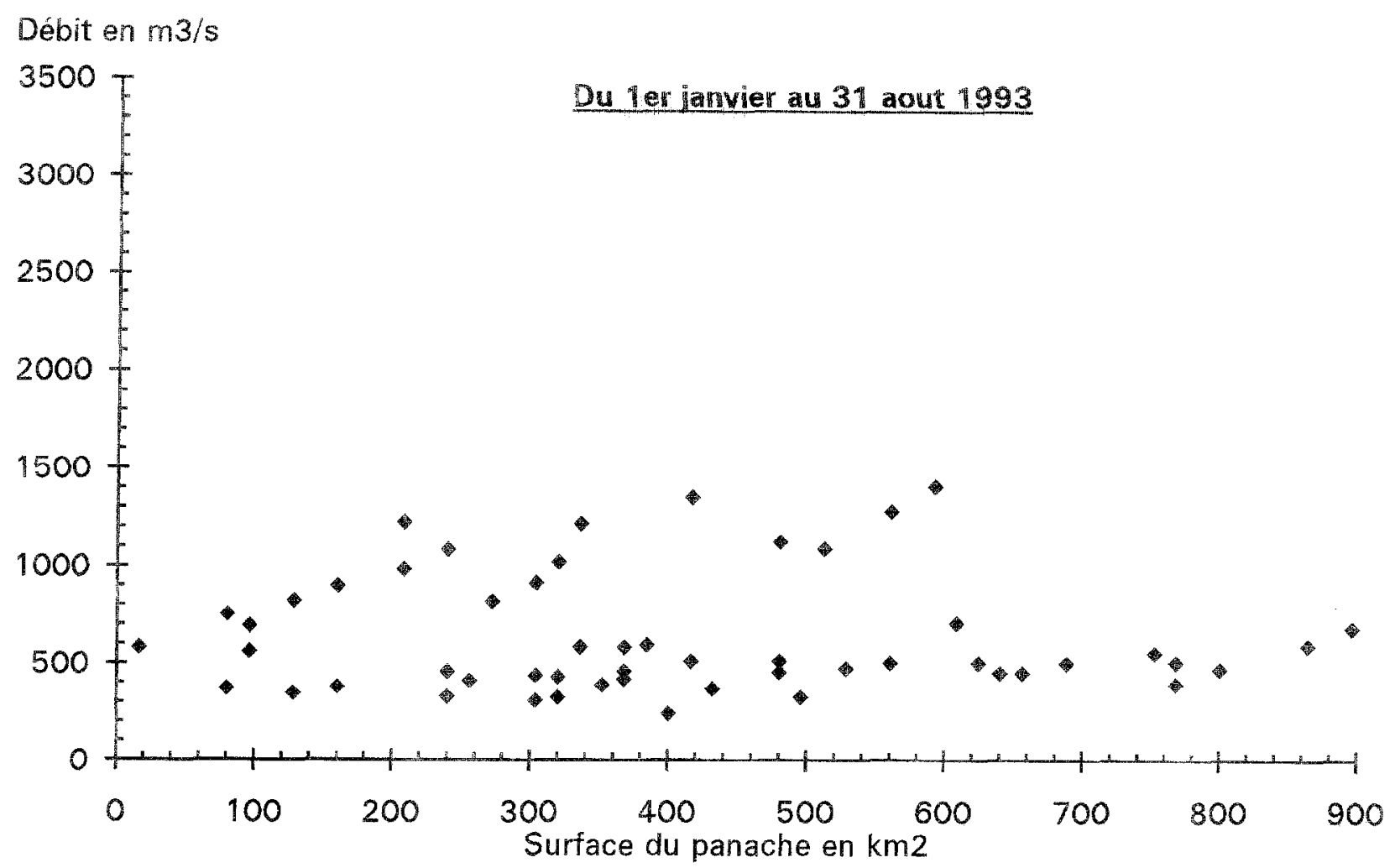

Figure 9. Variations de la surface du panache par rapport au débit fluvial du ler janvier an 31 août 1993.

Figure 9. Variations of the turbid plume areas in terms of fluvial outputs from 1 January to 31 August 1993.

sion. Deux situations typiques se répètent le plus souvenć. En période de faible débit (étiage) pendant la mission Calibsat-4 par exemple, le panache de surface est réduit à l'embouchure. Le panache de fond est plus étendu (figure 6). En période de fort débit (crue), pendant la mission Calibsat-3, le panache de surface est bien marqué. Il s'étend jusqu'à $2^{\circ} 20^{\prime}$ ouest. Le panache de fond s'étend encore plus loin jusqu'à $2^{\circ} 40^{\prime}$ ouest (figure 7 ).

Ainsi il existe généralement deux panaches turbides issus de l'estuaire : a) un panache de surface dont l'épaisseur varie entre $15 \mathrm{~m}$ (toute la tranche d'eau) à la sortie de l'embouchure, et $5 \mathrm{~m}$ au large, mais la limite inférieure de ce panache est peu marquée et souvent invisible sur les enregistrements du turbidimètre; b) un panache de fond, plus étendu que celui de surface, où les turbidités sont souvent plus élevées au-dessus des vasières ouest et sud Girondc. Il est très possible que ce panache de fond ait deux origines : remise en suspension de vases au dessus des vasières et apport direct par la Gironde. Les deux néphélö̈des se mélangeant, il n'est pas possible de définir sur les enregistrements les parts respectives de leurs deux. sources.

\subsection{Variations du panache de surface en fonction des débits fluviaux}

Pour étudier ces variations nous disposons des données AVHRR (basse résolution) et des données de débit fluvial «Garonne + Dordogne ». Les surfaces du panache de surface ont été mesurées sur les images C1 de l'AVHRR en comptant le nombre des pixels $\left(1\right.$ pixel $\left.=16 \mathrm{~km}^{2}\right)$ de réflectance élevée en continuité avec l'embouchure.

En 1992, deux périodes de forts débits se produisent en juin $\left(4850 \mathrm{~m}^{3} \cdot \mathrm{s}^{-1}\right.$ le 13 juin) et en octobre-novembre (3398 $\mathrm{m}^{3} . \mathrm{s}^{-1}$ le 7 octobre, $3329 \mathrm{~m}^{3} . \mathrm{s}^{-1}$ le 2 novembre, $3308 \mathrm{~m}^{3} \cdot \mathrm{s}^{-1}$ le 15 novembre et $4359 \mathrm{~m}^{3} . \mathrm{s}^{-1}$ le 4 décembre). Les surfaces du panache (série discontinue, 57 images) dépassent rarement $800 \mathrm{~km}^{2}$. Les surfaces les plus grandes apparaissent le 20 février $\left(832 \mathrm{~km}^{2}\right)$, le 9 avril 


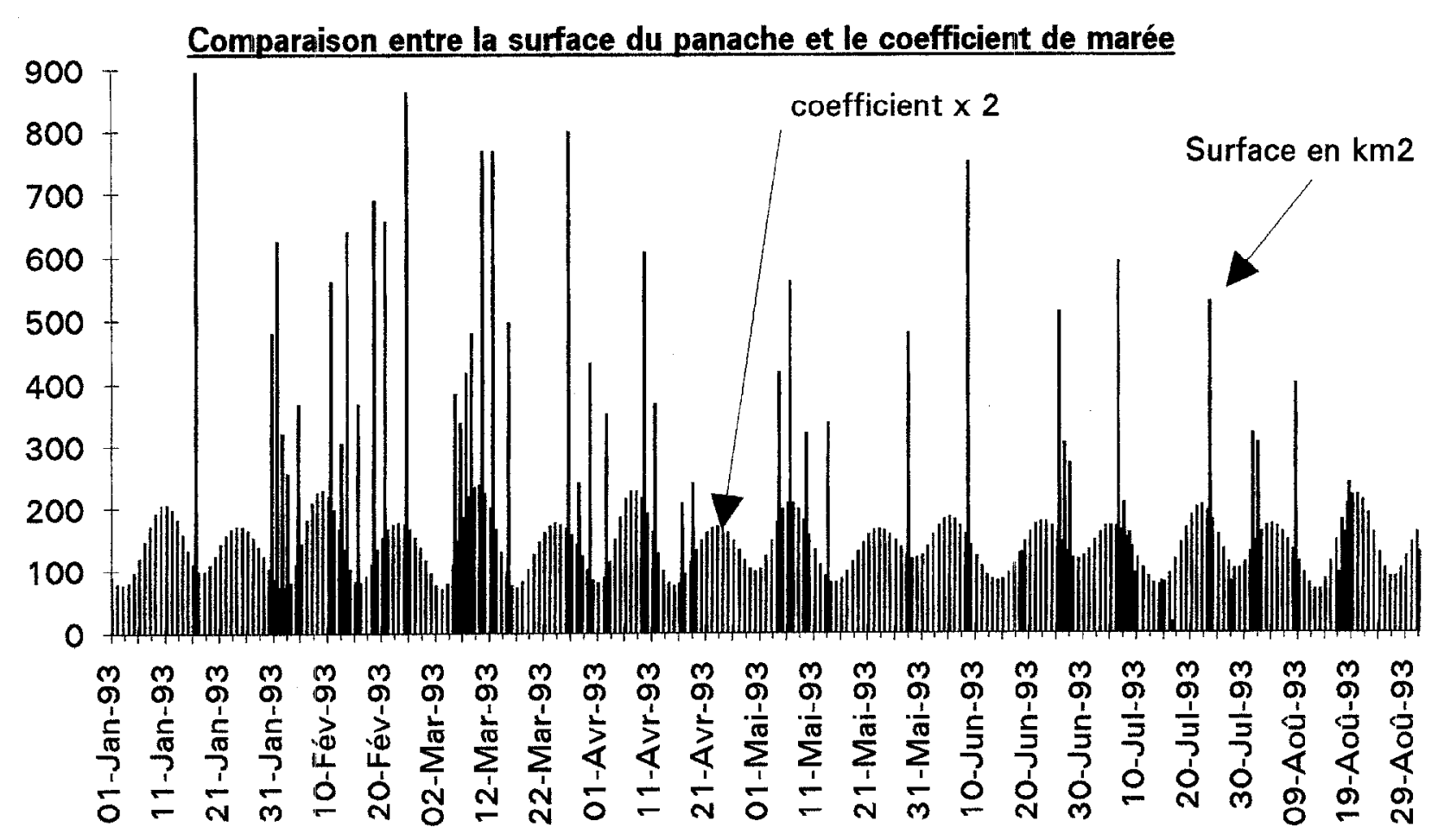

Figure 10. Superposition du cycle mortes-eaux, vives-eaux et des surfaces du panache vues par l'AVHRR du $1^{\text {er }}$ janvier au 31 août 1993.

Figure 10. Superposition of the neap and spring tide cycle and of the turbid plume areas from the AVHRR data from 1 January to 31 August 1993.

$\left(960 \mathrm{~km}^{2}\right)$, le 7 juillet $\left(880 \mathrm{~km}^{2}\right)$, le 10 septembre $\left(864 \mathrm{~km}^{2}\right)$, le $1^{\text {er }}$ novembre $\left(1008 \mathrm{~km}^{2}\right)$ et le 23 novembre $\left(784 \mathrm{~km}^{2}\right)$. Les surfaces les plus faibles apparaissent durant le mois août. Une augmentation importante du panache apparaît peu après les crues de début juin et d'automne. Mais les surfaces les plus faibles sont situées en période d'étiage. Cette faible concordance est illustrée sur le diagramme de la figure 8 qui représente les variations du débit (axe des Y) par rapport aux surfaces du panache (axe des X).

De janvier à fin août 1993, le scénario est différcnt avcc une crue fin avril, le $29,\left(3465 \mathrm{~m}^{3} \cdot \mathrm{s}^{-1}\right)$ suivie de débits moyens en juin et de débits très faibles en août. Les surfaces les plus grandes sont observées le 16 janvier $\left(896 \mathrm{~km}^{2}\right)$, le 24 février $\left(864 \mathrm{~km}^{2}\right)$ et le 26 mai $\left(800 \mathrm{~km}^{2}\right)$ et les surfaces les plus faibles en juillet et août. Ainsi ces variations de déhit semblent sans effet (mais la série est discontinue, 53 images) sur les surfaces du panache, sauf pour la période estivale. De même, lorsque les surfaces du panache sont reportées en fonction des débits, (figure 9), les points sont dispersés. Nous avons vérifié si cette absence de concordance ne provenait pas des délais de transit entre l'entrée dans la Gironde et la sortie sur le plateau continental, $100 \mathrm{~km}$ plus loin, délais estimés par Allen [1] et Castaing [6]. Ces auteurs donnent des transits de l'ordre de 4 à 5 jours pour les crues à plus de 50 jours pour les étiages, et 20 jours pour les débits moyens. Même en tenant compte de ces décalages le résultat est identique au précédent. Seule une relation saisonnière semble exister puisqu'en 1992 et en 1993, les surfaces d'extension du panache sont faibles en été et plus étendues en hiver et au printemps.

\subsection{Variations du panache de surface en fonction de la marée}

La marée peut agir de deux façons, par son cycle semidiurne et par l'alternance des mortcs-caux ct des viveseaux.

\subsubsection{Cycle semi-diurne}

Des vues aériennes prises à intervalles de $3 \mathrm{~h}$ ont montré le déplacement et l'extension des fronts turbides vers le large au cours du jusant [5]. Mais le passage du satellite à 


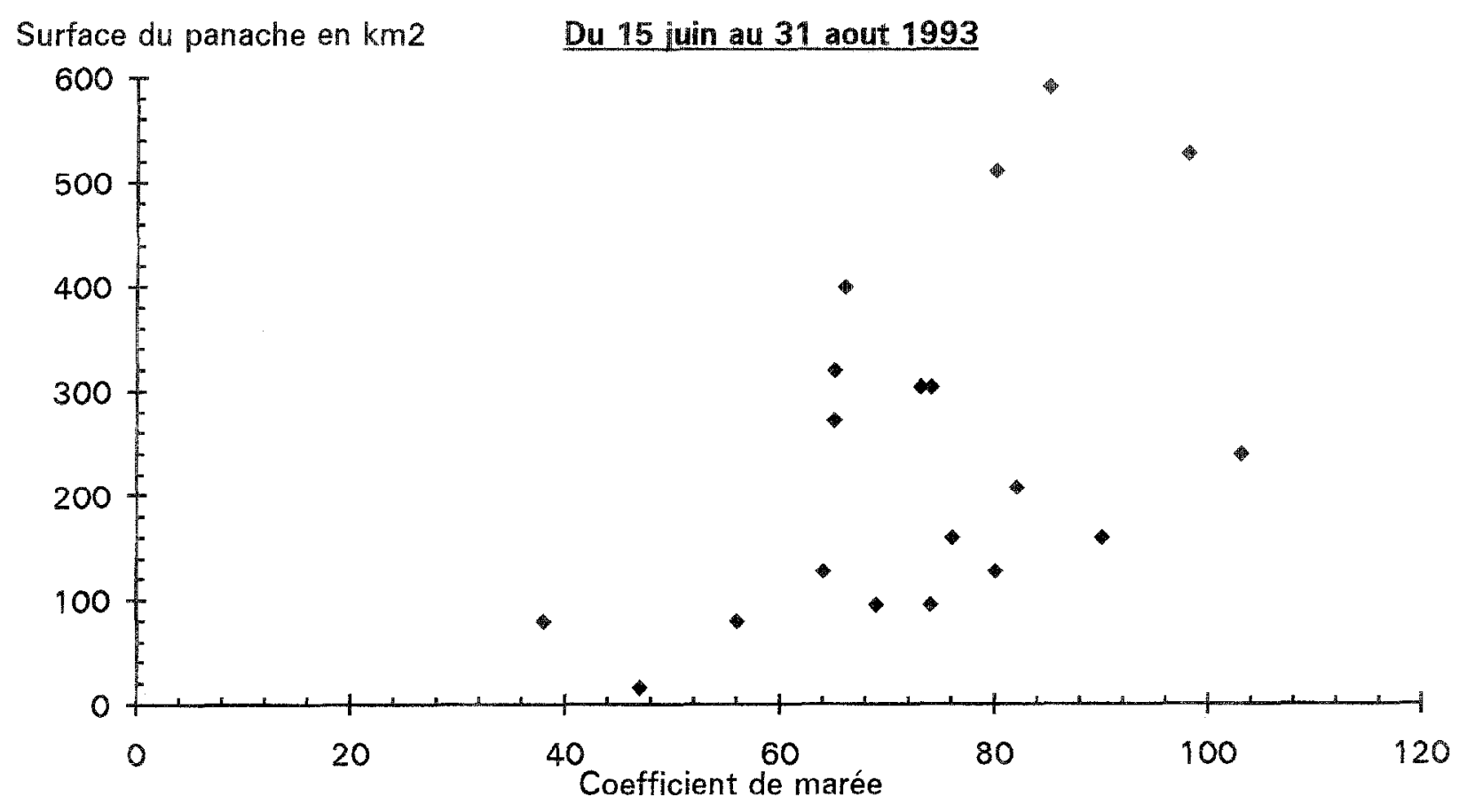

Figure 11. Variations de la surface du panache en fonction des coefficients de marée en période d'étiage en 1993.

Figure 11. Variations of the turbid plume areas in terms of tidal coefficients during a low water period in 1993.

intervalles de $24 \mathrm{~h}$ ne permet pas une analyse précise des effets de la marée. Nous avons comparé des images AVHRR prises à des moments différents de la marée, sans tenir compte des dates. Les résultats obtenus montrent la présence continuelle du panache quelle que soit l'heure de la marée. Par conséquent, les particules en suspension ne quittent pas rapidement la couche de surface, tout au moins pour une partie d'entre elles. Le panache expulsé en jusant s'étend vers le large durant le flot suivant.

\subsubsection{Cycle des vives-eaux et des mortes-eaux}

Les coefficients de marée sont d'autant plus élevés que l'amplitude de la marée est importante (le coefficient de marée est défini par le rapport « amplitude de la marée du jour $\gg / \ll$ amplitude de la plus grande marée, multiplié par $120 »$ ). Plus l'amplitude est importante (période de viveseaux), plus les courants sont élevés dans l'estuaire. Sur la figure 10, correspondant à 1993, la courbe de variation des coefficients de marée est superposée aux variations de surface du panache. D'une façon générale, les surfaces sont plus grandes en phase de vives-eaux et durant les périodes allant des vives-eaux aux mortes-eaux (périodes de déchets). Cette relation est également perceptible sur le diagramme de la figure 11 montrant les variations de surface du panache en fonction des coefficients en période d'étiage. Ce résultat s'explique dans la mesure où le débit tidal est 50 à 100 fois plus élevé que le débit fluvial. En effet les volumes entrant et sortant de l'estuaire sous l'effet de la marée seule varient entre $92600 \mathrm{~m}^{3} . \mathrm{s}^{-1}$ en période de vives-eaux et $51000 \mathrm{~m}^{3} . \mathrm{s}^{-1}$ en période de mortes-eaux [3] alors que le débit fluvial est de l'ordre du millier de $\mathrm{m}^{3} \cdot \mathrm{s}^{-1}[1]$. Mais cette relation n'est pas systématique. Il existe aussi des périodes où le panache est étendu en mortes-eaux. Seul un modèle numérique intégrant les paramètres hydrologiques pourrait apporter une réponse satisfaisante.

\subsection{Variations du panache de surface en fonction du vent}

La circulation du proche plateau continental est complexe car soumise à de multiples facteurs variant tantôt brutalement (vent) tantôt progressivement (température) et de façon imprévisible (crues, tempêtes). C'est pourquoi, un 


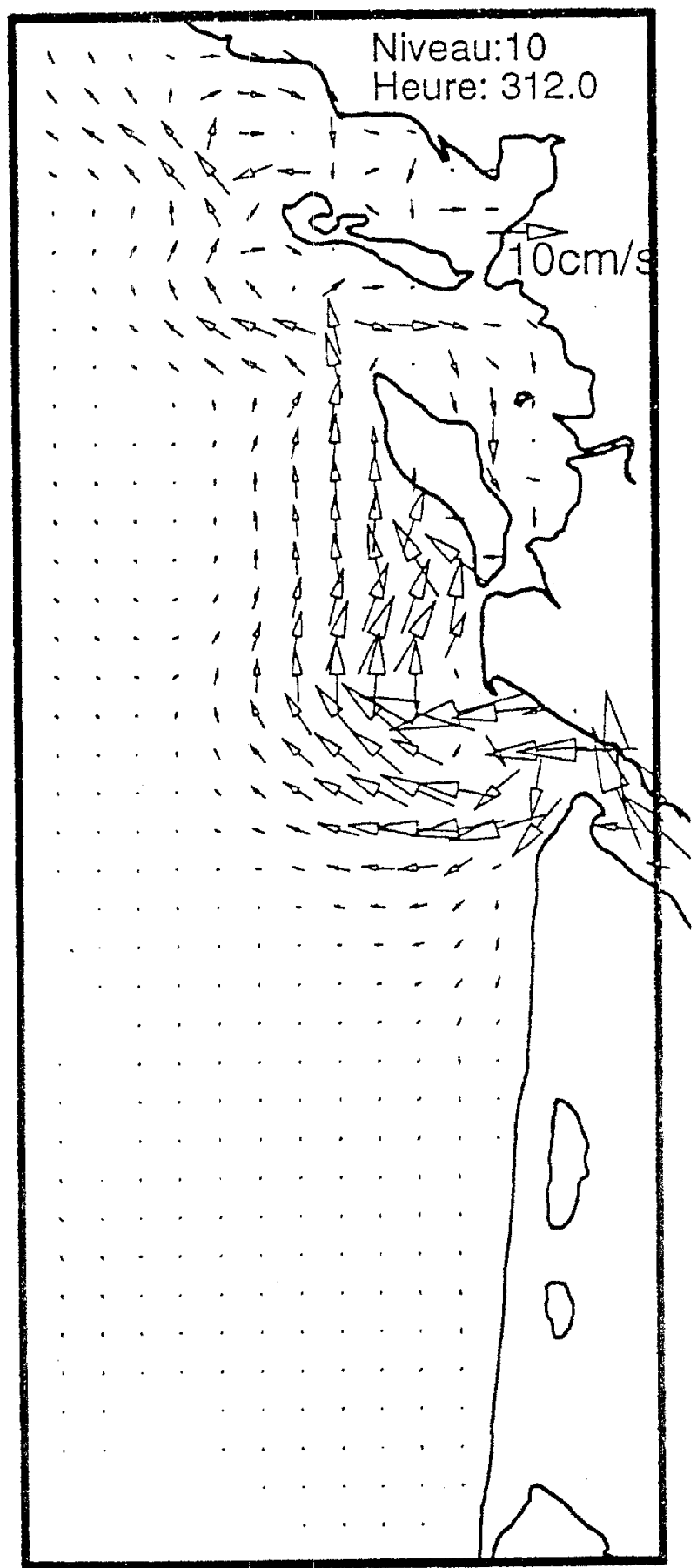

Figure 12. Simulation des courants de surface engendrés par une crue de $3000 \mathrm{~m}^{3} \cdot \mathrm{s}^{-1}$, sans vent, d'après le modèle hydrodynamique d'Ifremer.

Figure 12. Speed current vectors induced by a Gironde flood (3000 $\mathrm{m}^{3} \cdot \mathrm{s}^{-1}$ ) without wind, from the Ifremer hydrodynamic modelisation.
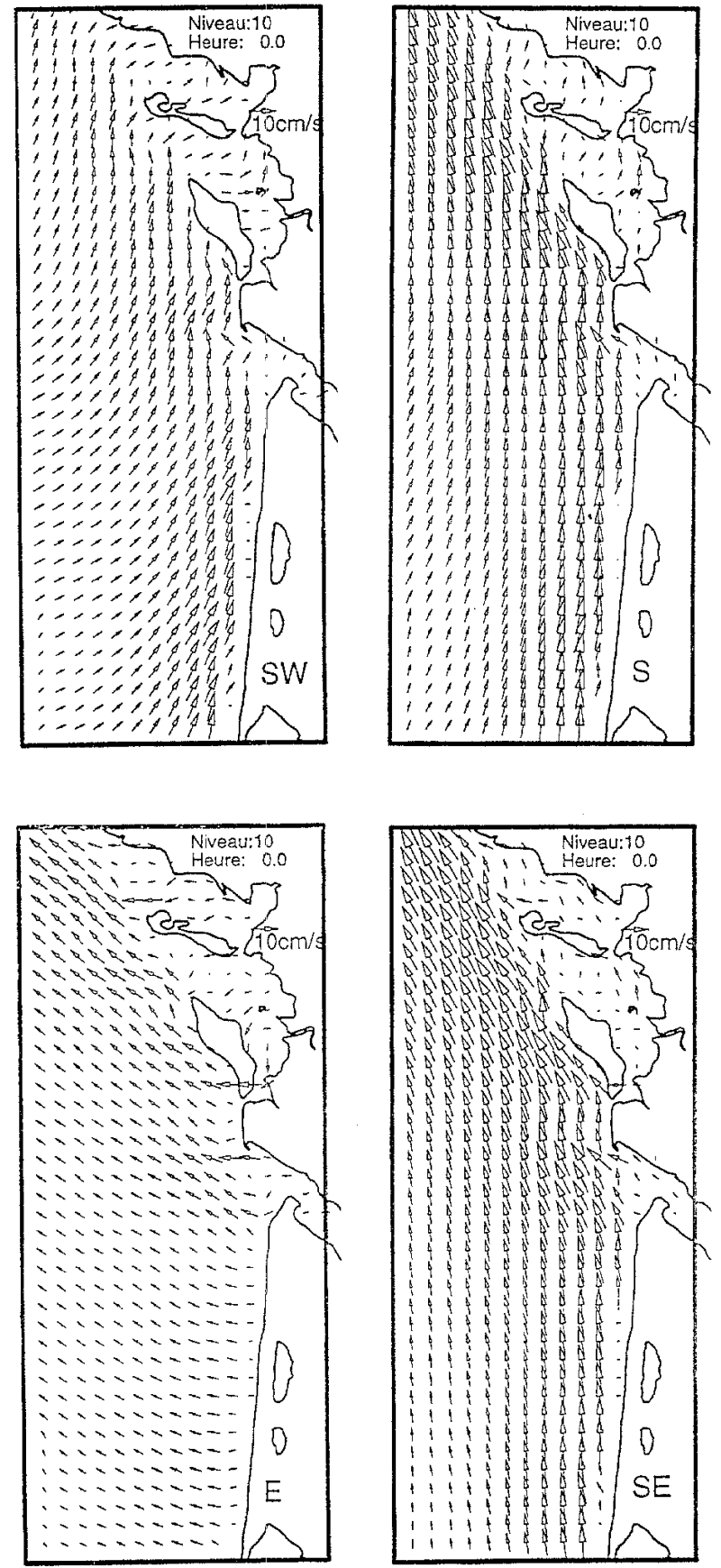

Figure 13. Courants de surface dirigés vers le nord induits par des vents de SW, S, E et SE d'après le modèle d'Ifremer

Figure 13. Surface currents oriented to the north, induced by SW, S, $\mathrm{E}$ and SE winds from the Ifremer modelisation.

modèle numérique 3D a été développé par IFREMER [14] dans le cadre du programme PNOC-Atlantique. $\mathrm{Ce}$ 

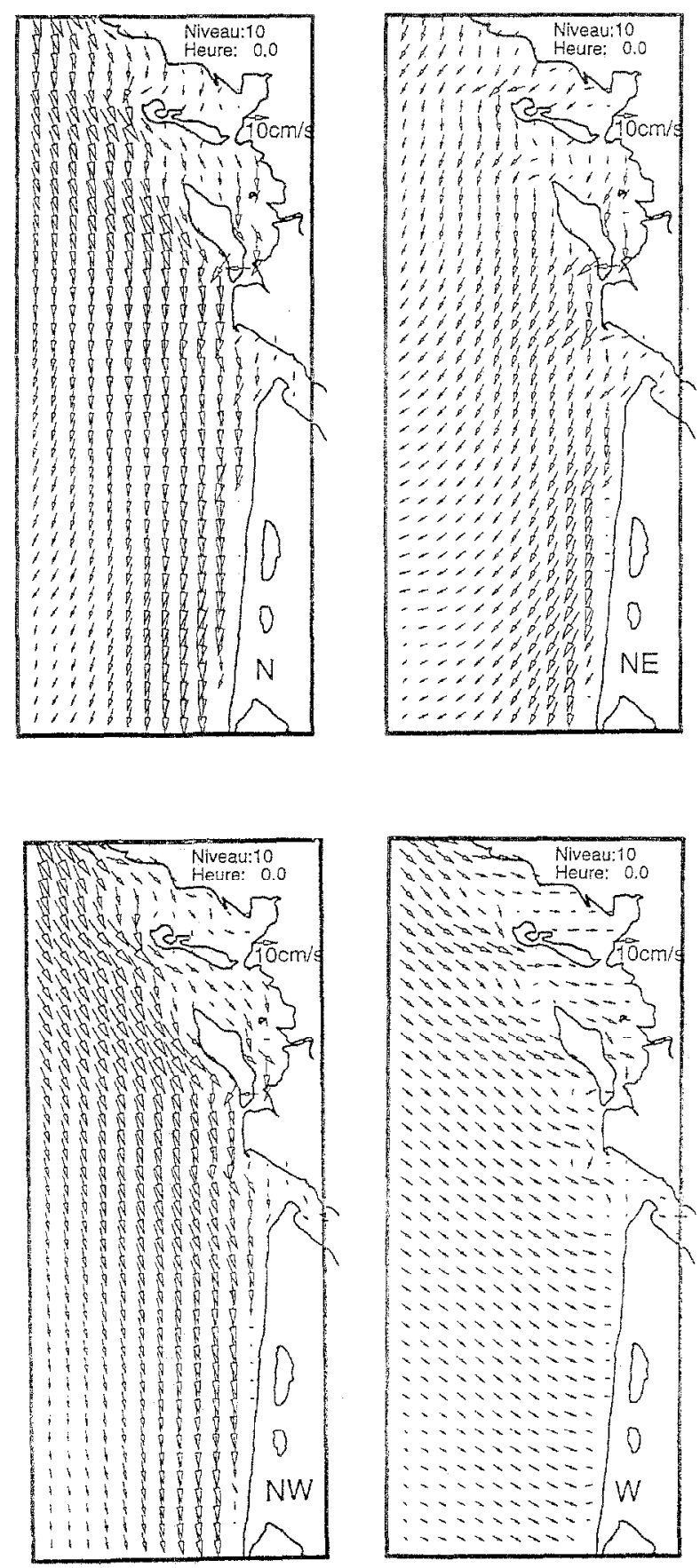

Figure 14. Courants de surface dirigés vers le sud, induits par des vents de $\mathrm{N}, \mathrm{NE}, \mathrm{NW}$ et $\mathrm{W}$, d'après le modèle d'Ifremer.

Figure 14. Surface currents oriented to the south, induced by N, NE, $\mathrm{NW}$ and $\mathrm{W}$ winds from the Ifremer modelisation.

modèle prend en compte les débits fluviaux, les courants de marée, la variation de température et le vent. Afin d'interpréter les orientations du panache girondin, plusieurs simulations courantologiques ont été réalisées pour des vents de directions différentes et en l'absence de vent.

La simulation (figure 12) représente la circulation induite par 28 jours d'un débit de $3000 \mathrm{~m}^{3} \cdot \mathrm{s}^{-1}$ en l'absence de vent et où seule la marée résiduelle est prise en compte. Elle fait apparaître un courant de densité orienté vers l'ouest, puis vers le nord sous l'effet de la force de Coriolis. Ce courant longe l'île d'Oléron. Ensuite, une branche pénètre dans le Pertuis d'Antioche et une autre branche poursuit son chemin vers lc nord, induisant un contrecourant orienté vers le sud à l'abri des îles de Ré et d'Oléron.

Par vent constant soufflant avec une vitesse de $10 \mathrm{~cm} . \mathrm{s}^{-1}$, et en l'absence de débit estuarien, huit cas typiques peuvent être présentés. Pour un vent de sud-ouest (figure 13, SW) le courant de surface est orienté vers le nord-est au large et vers le nord le long du littoral. Les vitesses augmentent le long de la côte du Médoc ê au nurd de l'embouchure. Par vent du sud (figure 13, S), le courant est orienté vers le nord. Il est beaucoup plus élevé près du littoral qu'au large. Par vent d'est (figure 13, E) les courants de surface sont faibles ct oricntés vers le nord-ouest. Par vent de sud-est, (figure 13, SE) les courants sont orientés vers le nord, avec des courants élevés au nord de la Gironde. Par vent du nord (figure 14, N), les courants de surface sont orientés vers le sud. Par vent du nord-est (figure 14, NE), les courants sont nettement plus faibles, orientés vers le sud le long de la côte et vers le sud-ouest au large. Par vent de nord-ouest (figure 14, NW), les courants de surface orientés vers le sud, longent la côte. Ils sont élevés au nord de l'embouchure. Par vent d'ouest (figure 14, W) les courants sont faibles et orientés vers le sud-est.

L'observation des images AVHRR à basse résolution permet de distinguer au moins six types d'orientation du panache schématisés sur la figure 15 : type 1) panache orienté vers le nord; type 2) panache orienté vers le NW ou WNW; type 3) panache orienté vers l'ouest; type 4) panache réduit à l'embouchure; type 5) panache double avec parties nord et sud; type 6) panache orienté vers le sud. Pour chacun de ces types, le nombre d'observations en 1992 et 1993 est répertorié dans le tableau II.

Parmi les 110 images exploitables en 1992 et 1993 (57 et 53 respectivement), les panaches sont en majorité (34 observations) orientés vers le NW ou WNW. Ces orientations correspondent à celles des courants de den-sité simulés précédemment (figure 12). Les vents de sec- 


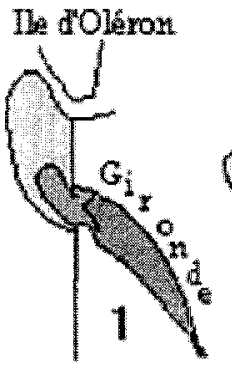

Nord

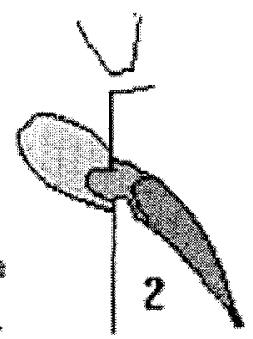

N.W.

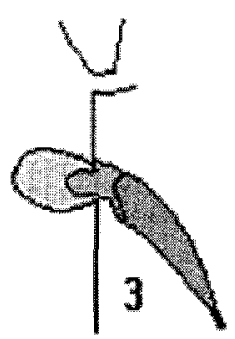

Ouest

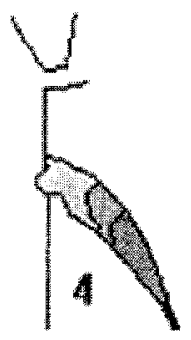

Réduit

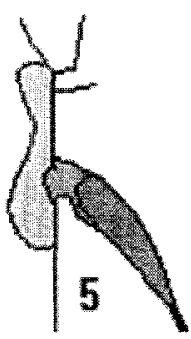

Double

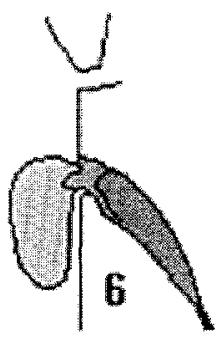

Sud

Figure 15. Principales orientations du panache de la Gironde d'après les données AVHRR de 1992 et 1993. Les intensités de griș augmentent avec la turbidité.

Figure 15. Main orientations of the Gironde turbid plume from the AVHRR data in 1992 and 1993.

Tableau II. Orientations du panache de la Gironde en 1992 et 1993.

Table II. Plume orientations of the Gironde in 1992 and 1993.

\begin{tabular}{lcc}
\hline Types & Orientations en 1992 & Orientations en 1993 \\
\hline 1- Panache orienté vers le Nord & 5 & 2 \\
2- Panache orienté vers le NW ou W.N.W. & 17 & 17 \\
3- Panache orienté vers l'Ouest & 12 & 10 \\
4- Panache réduit à l'embouchure & 9 & 13 \\
5- Panache double avec parties Nord et Sud & 1 & 3 \\
6- Panache orienté vers le Sud ou S.S.W. & 13 & 8 \\
\hline
\end{tabular}

teur nord (de NE à NW, voir WNW) sont responsables des panaches orientés vers le sud (21 observations) après la modélisation (figure 13). Les panaches orientés vers le nord ( 7 observations) sont déformés et entraînés soit par la force de Coriolis, soit par des vents du secteur SW, (de sud à WSW) d'après le modèle d'IFREMER (figure 14). Les vents de secteur est seraient responsables des panaches orientés vers l'ouest ( 22 observations). Les panaches réduits à l'embouchure (type 4) n'ont pas d'orientation visible car ils ne se développent pas vers le large (22 observations). Ils correspondent aux périodes d'étiage. Les panaches doubles (type 5) sont relativement rares (4 observations). La faible résolution de ces images ne permet pas de dire si ces panaches correspondent à deux expulsions distinctes de la Gironde, ou si ces panaches proviennent du pertuis de Maumusson et de la Gironde, tous les deux orientés vers le sud.

Ainsi ces observations mettent en évidence l'importance du vent sur l'orientation du panache de surface de la
Gironde. Des observations similaires ont porté sur d'autres panaches de surface, tel que ceux du Mississippi [22] ou du Rhône [9].

\section{CONCLUSION}

Ces recherches effectuées dans le cadre du PNOC apportent quelques précisions sur l'exploitation des images AVHRR/NOAA pour cartographier et quantifier les plumes turbides dans les zones côtières. Le traitement utilisé pour comparer les images satellitales est basé sur le concept des « eaux claires ». La réflectance la plus faible, sur des eaux du large proches du secteur mais pauvres en MES, est considérée comme le signal atmosphérique. Elle est soustraite de l'image. Un traitement plus précis tenant compte de la transmission diffuse peut être appliqué dans des cas ponctuels. Lorsque le scintillement solaire est élevé, il est possible d'utiliser la soustraction des images $\mathrm{C} 1-\mathrm{C} 2$ qui permet de voir l'extension du pana- 
che turbide, mais les données sont faussées puisque les turbidités ont aussi un effet sur la bande spectrale du proche infrarouge (canal C2).

Par comparaison des réflectances AVHRR aux concentrations en MES enregistrées au cours de plusicurs missions océanographiques, des relations apparaissent assez clairement: Elles sont linéaires pour les faibles turbidités (audessous de $15 \mathrm{mg} . \mathrm{l}^{-1}$ environ), mais aux turbidités plus élevées, la relation devient logarithmique. Les résultats sont confirmés par des mesures réalisées avec un radiomètre de terrain. La pente des droites d'ajustement varie d'une mission à l'autre dans des proportions parfois importantes. Ces variations, qui reflètent probablement un changement dans la composition ou dans la taille des particules, montrent l'importance des mesures in situ.

L'étude du panache de la Gironde à partir d'une centaine d'images satellitales AVHRR à basse résolution montre que celui-ci a une extension relativement réduite en dehors des périodes de crues. Sa surface est souvent comprise entre $200 \mathrm{~km}^{2}$ et $600 \mathrm{~km}^{2}$. Il n'a pas été possible de mettre en évidence une relation nette entre les variations de débit et les extensions du panache, celles-ci étant plutôt liées aux marées. En revanche, après les très fortes crues de janvier 1994, l'extension est bien perceptible.
Dans la plupart des cas, elle est plus importante pendant et juste après une marée de vives-eaux (période de déchet). La forme du panache et son orientation semblent dépendre du vent, sauf lors d'une expulsion importante engendrant un courant de densité ; dans ce cas la plume est expulsée vers le nord-ouest. En été le panache est réduit, ou orienté vers le sud à cause des vents dominants de secteur nord; en hiver, il est orienté vers le NW et le nord à cause du courant de densité ou des vents d'ouest dominants.

\section{Remerciements}

Cette étude a été réalisée grâce aux soutiens du Programme National d'Océanographie Côtière (PNOC) et du Programme National de Télédétection Spatiale (PNTS). Les auteurs adressent leurs plus vifs remerciements aux responsables de ces programmes, en particulier au coordinateur du secteur « Atlantique », P. Laborde, ainsi qu'aux équipages des navires océanographiques, à $\mathrm{H}$. LeGléau du Centre de Météorologie Spatiale (CMS) de Lannion et à R. Santer, de l'Université du Littoral Côte d'Opale.

\section{RÉFÉRENCES}

[1] Allen P., Étude des processus sédimentaires dans l'estuaire de la Gironde, Mém. Inst. Géol. Bassin Aquitaine, n 5, (1973) $313 \mathrm{p}$.

[2] Berne S., Castaing P., Le Drezen E., Lericolais G., Morphology, internal structure and reversal of asymmetry of large subtidal dunes in the entrance to Gironde estuary (France), I. Sediment Petrol. 63 (5) (1996) 780-793.

[3] Bonnefille R. Étude de l'aménagement de l'estuaire de la Gironde. Rapport $n^{\circ} 9$. Étude synthétique du régime hydraulique, Lab. Nat. Hydraulique, Chatou (1970) HC042 R411 690 DHM.

[4] Bukata R.P., Jerome J.H., Burton J.E., Particulate concentrations in lake St Clair as recorded by a shipborne multispectral optical monitoring system, Remote Sens. Environ. 25 (1988) $201-229$.

[5] Castaing P., Allen G.P., Houdart M., Moign Y., Étude par télédétection de la dispersion en mer des eaux estuariennes issues de la Gironde et du Pertuis de Maumusson, Oceanol. Acta 2 (4) (1979) 459-468.

[6] Castaing P., Le transfert à l'océan des suspension estuariennes. Le cas de la Gironde, Thèse d'Etat, Univ. Bordeaux-I. (1981) $530 \mathrm{p}$.
[7] Dilligeard E., Télédétection des eaux du cas II. Caractérisation des sédiments marins, Thèse de l'Université du Littoral Côte d'Opale, Dunkerque (1997) 184 p.

[8] Eisma D., Particle size of suspended matter in estuaries, GeoMarine Letters 11 (1991) 147-153.

[9] Estournel C., Kondrachoff V., Marsaleix P., Vehil R., The plume of the Rhonc: numcrical simulation and remote sensing, Cont. Shelf Res. 17 (1997) 889-924.

[10] Froidefond J.M., Castaing P., Mirmand M., Ruch P., Analysis of the turbid plume of the Gironde estuary (France) based on SPOT radiometric data, Remote Sens. Environ. 36 (1991) 149-164.

[11] Froidefond J.M., Castaing P., Jouanneau J.M., Prud'homme R., Dinet A., Method for the quantification of suspended sediments from AVHRR NOAA-11 satellite data, Int. J. Remote Sens. 14 (5) (1993) 885-894.

[12] Froidefond J-M., Castaing P., Jouanneau J.M., Distribution of suspended matter in a coastal upwelling area. Satellite data and in situ measurements, J, Marine Syst. 8 (1996) 91-105.

[13] Hermida J.E., Dispersion des eaux estuariennes de la Gironde sur le plateau continental, Thèse de l'Université Bordeaux $\mathbb{i}$, $\mathrm{n}^{\circ} 1683$ (1997) $178 \mathrm{p}$. 
[14] Jegou A-M., Lazure P., Quelques aspects de la circulation sur le plateau Atlantique, in: Actes du IV ${ }^{\text {eme }}$ Colloque International du Golfe de Gascogne, (eds.) Cendrero et Olaso. Santander, 12-14 avril 1994 (1994) 99-106.

[15] Laborde P., Chantier Atlantique. Bilan des actions entreprises et synthèse des principaux résultats obtenus au terne des quatres premières années du programme. Rapport PNOC (1995) $24 \mathrm{p}$.

[16] Lin Rong Gen, Étude du potentiel de dégradation de la matière organique particulaire au passage eau douce - eau salée. Cas de l'estuaire de la Gironde, Thèse, Univ. Bordeaux I (1988) $209 \mathrm{p}$.

[17] Novo, E.M.L.M., Steffen C.A., Braga C.L.H., Results of a laboratory experiment relating spectral reflectance to total suspended solids, Remote Sens. Fnviron. 36 (1991) 67-72.

[18] Ouillon S., Forget Ph., Froidefond J.M., Naudin J.J., Estimating suspended matter concentrations from SPOT data and from field measurements in the Rhône river plume, MTS Journal 31 (2) (1997) 15-20.
[19] Ritchie J.C., Cooper C.M., Shiebe F.R., The relationship of MSS and TM digital data with suspended sediments, chlorophyll and temperature in Moon Lake, Mississipi, Remote Sens. Environ. 33 (1990) 137-148.

[20] Stumpf R.P., Pennock J.R., Calibration of a general optical equation for remote sensing of suspended sediments in a moderatly turbid estuary, J. Geophys. Res. 94 (C10) (1989) 14363-14371.

[21] Tanré D., Deroo C., Duhaut P., Herman M., Morcrette J.J., Perbos J., Deschamps P.Y., Simulation of the Satellite Signal in the Solar Spectrum (5S), L.O.A., Univ. de Lille (1987) $149 \mathrm{p}$.

[22] Walker N., Satellite assessment of Mississipi river plume variability: causes and predictability, Remote Sens. Environ. 58 (1996) 21-35.

[23] Weber O., Jouanneau J.M., Ruch P., Mirmand M., Grain size relationship between suspended matter originating in the Gironde estuary and shelf mud-patch deposits, Mar. Geol. 96 (1991) 159-165. 